\title{
Feld-Ahorn (Acer campestre), Baum des Jahres 2015
}

\author{
VEIT M. Dörken
}

\begin{abstract}
The field maple (Acer campestre, Sapindaceae) is native to Europe. This deciduous tree is characterized by a mostly corky bark. It is widely grown as an ornamental. There are many cultvars that differ in respect to foliage and overall appearance. The biology, ecology, and use of the tree are outlined. Acer campestre is the tree of the year 2015.
\end{abstract}

\section{Zusammenfassung}

Feld-Ahorn (Acer campestre, Sapindaceae) ist in Europa heimisch. Der laubwerfende Baum bildet häufig eine korkige Borke. Auch als Ziergehölz ist der Feld-Ahorn weit verbreitet. Es gibt viele Kultursorten, die sich in Habitus und Belaubung voneinander unterscheiden. Biologie, Ökologie und Nutzung des Feld-Ahorns werden erläutert. Acer campestre ist Baum des Jahres 2015 .

\section{Einleitung}

Bereits zweimal wurden heimische AhornArten zum Baum des Jahres ausgerufen: 1995 der Spitz-Ahorn und 2009 der Berg-Ahorn. Für dieses Jahr wurde der Feld-Ahorn (Acer campestre), der auch Maßholder genannt wird, ausgewählt. Das Motto zu diesem Jahresbaum lautet „Der kleine zarte Bruder - oft übersehen“. Aufgrund seines langsamen Wuchses, der vergleichsweise geringen Wuchshöhe, der Mehrstämmigkeit und des knorrigen Habitus alter Bäume ist dieser Ahorn relativ unauffällig. Er wird weithin als der „kleine Bruder" des Spitzund Berg-Ahorns bezeichnet. Da Feld-Ahorn nur selten bestandsbildend ist, sondern oft nur vereinzelt in lockeren Baumgruppen oder in Waldrändern vorkommt, wird er leicht übersehen. Auch wenn er in weiten Teilen Deutschlands recht häufig ist, trifft dies z. B. auf Teile Ostdeutschlands nicht zu. Im Berliner Raum gilt die Art als extrem selten und in Brandenburg wird sie sogar als gefährdet eingestuft (Prasse et al. 2001). Als Baum des Jahres soll dieser kleine Baum aber vor allem aufgrund ästhetischer, ökologischer und landschaftsgestalterischer Aspekte der breiten Öffentlichkeit ins Gedächtnis gerufen werden. Besonders die gärtnerischen Selektionen des Feld-Ahorns finden in der Gartenkultur, im Straßenraum sowie in der freien Landschaft vielfach Verwendung.

Abb. 1: Acer campestre, Habitus.

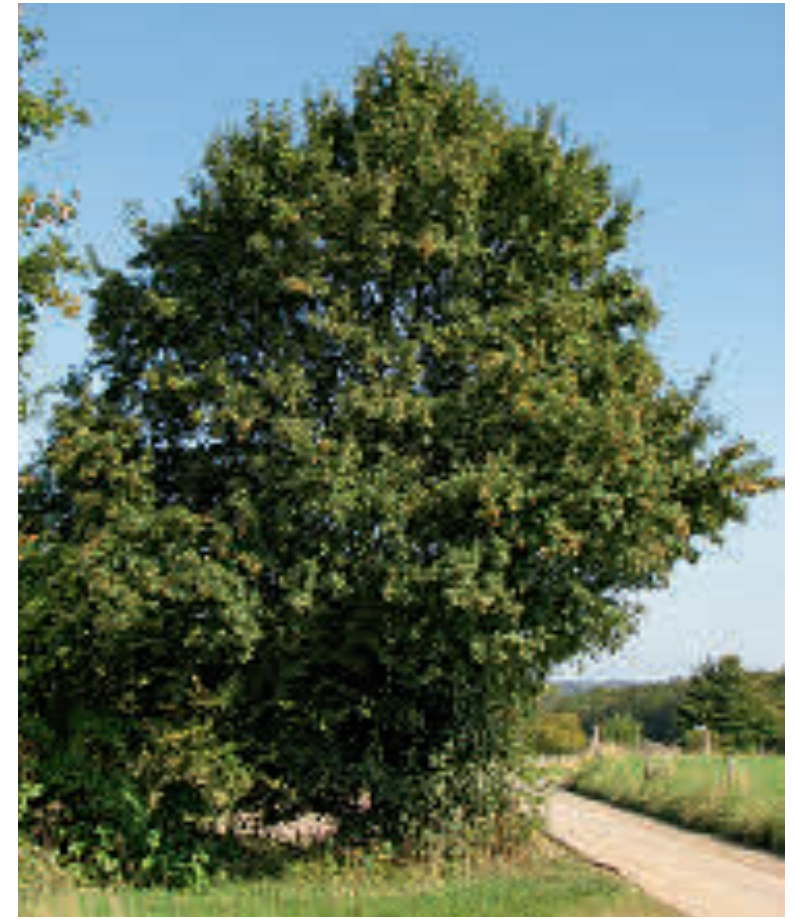

\section{Systematik}

Der Feld-Ahorn wurde früher zu den Ahorngewächsen (Aceraceae) gezählt. Nach neueren molekulargenetischen Erkenntnissen gehört die Gattung Acer nun aber der Familie der Seifenbaumgewächse (Sapindaceae) an. Hierzu gehört z. B. auch die Rosskastanie (Aesculus spp., ehemals Hippocastanaceae). Die Gattung Acer ist mit 124 Arten und einer großen Anzahl von beschriebenen Unterarten und Varietäten sehr formenreich und wird daher in 16 Sektionen unterteilt, diese wiederum in 19 Reihen (vAN Gelderen et al. 1994). Acer campestre gehört zur Sektion Platanoidea, zu der unter anderem 

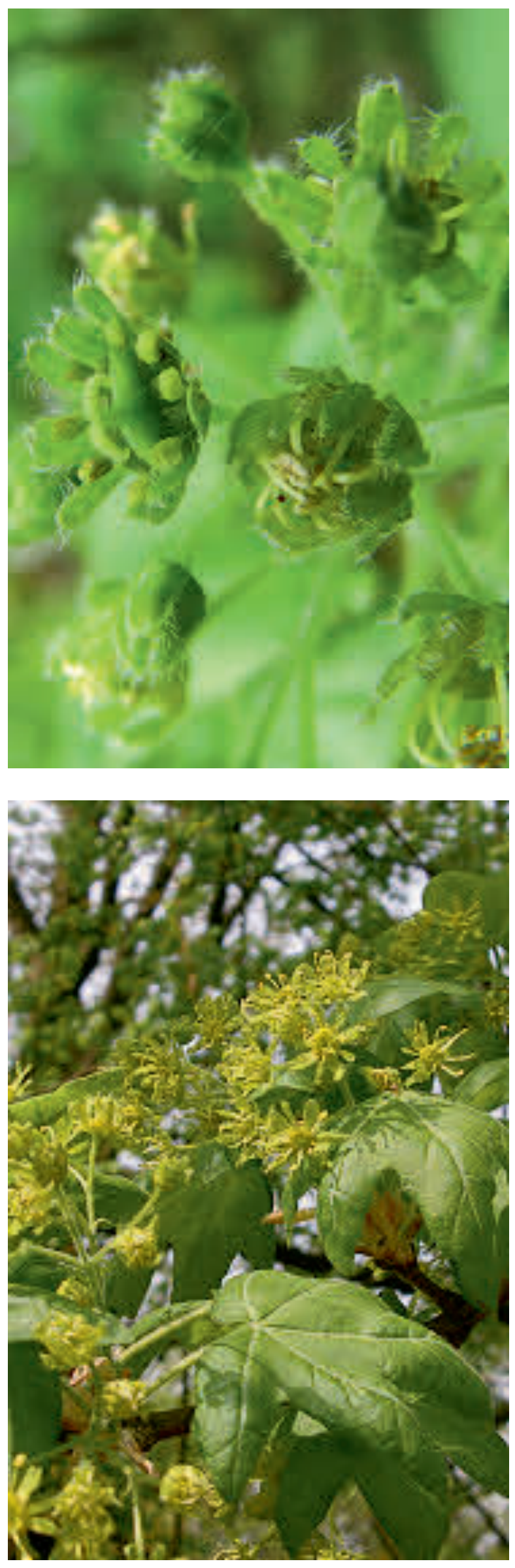

auch der heimische Spitz-Ahorn (Acer platanoides) gehört (vAN GELDERn et al. 1994). Wegen seines großen Verbreitungsgebietes ist auch die Formenvielfalt enorm. In der Vergangenheit wurden aufgrund habitueller Unterschiede, der Ausbildung bzw. des Fehlens von Korkleisten oder auch der Blattform zahlreiche Varietäten beschrieben. Von diesen werden heute aber nur noch wenige als taxonomisch relevant akzeptiert. Eine besonders bemerkenswerte Varietät ist Acer campestre var. acuminatilobum. Sie wurde in den 1940er-Jahren von Joseph PApp im ungarischen Mátra-Gebirge entdeckt (vaN Gelderen et al. 1994). Durch ihre spitzen Lappen unterscheiden sich die Blätter deutlich von den rundlappigen der var. campestre. Bevor diese Varietät als lebende Pflanze entdeckt wurde, waren ähnliche Blattformen schon aus fossilen Ablagerungen bekannt (van Gelderen \& van Gelderen 1999).

\section{Verbreitung}

Der Feld-Ahorn ist von Mitteleuropa bis WestRussland und Nord-Iran im Osten und von Südost-Europa bis Nord-Afrika verbreitet. Der Verbreitungsschwerpunkt liegt dabei in submediterranen bis subatlantischen Klimabereichen (Sснütт et al. 2002). Der Feld-Ahorn fehlte ursprünglich im niedersächsischen Tiefland und in Holland, ist aber heutzutage, wie auch Spitz- und Berg-Ahorn, in ganz Deutschland anzutreffen. Denn alle drei Ahorn-Arten werden häufig angepflanzt und verwildern dabei. Der Feld-Ahorn kommt bevorzugt in warmen Ebenen und Tälern vor. Im Bergland ist er trotzdem noch bis etwa 1000 m Höhe häufig anzutreffen (PIRC 1994). Seine Standortamplitude ist recht groß. Er kommt in Feldgehölzen, Heckenlandschaften, lichten Baumgruppen und auf offenen, besonnten Standorten vor. Feld-Ahorne findet man überwiegend in krautreichen Eichen-Hainbuchen-Wäldern (KIERMEIER 1993), dann allerdings meist im Waldmantel und nicht im Bestand selbst, da sie

Abb. 2 (oben): Auschnitt aus dem Blütenstand.

Abb. 3 (unten): Blühender Zweig mit jungen Blättern. 
sonnige bis halbschattige Standorte zum optimalen Gedeihen benötigen. Sie wachsen aber auch in Hartholzauen außerhalb des regelmäßigen Überschwemmungsbereiches sowie auf sehr trockenen, nährstoffarmen Dünenstandorten. Dabei werden mäßig feuchte bis frische, sandige Lehme mit einer basischen Reaktion sauren Standorten vorgezogen (Kiermeier 1993).

\section{Morphologie}

\subsection{Habitus}

Der Feld-Ahorn ist ein kleiner, meist 10-15 m hoher rundkroniger, langsamwüchsiger Baum. Im Alter kann er einen Stammdurchmesser von $1 \mathrm{~m}$ erreichen. Seine dunkelbraune Borke ähnelt mit ihrer kleinen rechteckigen Felderung derjenigen von Birnbäumen. Junge Sprossachsen sind zunächst an ihrer Spitze weißlich behaart, verkahlen jedoch rasch. Die zahlreichen Lentizellen sind hellbeige gefärbt und unauffällig. An älteren Seitentrieben sind mehr oder weniger starke Korkbildungen, mitunter auch breite Korkleisten, ausgeprägt. Die dunkel- bis rotbraunen Winterknospen sind klein und spitz und im Vergleich zu anderen Ahorn-Arten recht unauffällig. An ihrer Spitze sind sie weißlich behaart. Die Seitenknospen sind unauffällig klein und der Sprossachse angedrückt, an starkwüchsigen Trieben stehen sie schräg ab. Der Feld-Ahorn ist ein intensiver Herzwurzler mit einem hohen Anteil an Faserwurzeln im oberen Bodenhorizont. Daneben werden zahlreiche kräftige Senkwurzeln ausgebildet (SсHÜтT et al. 2002).

\subsection{Belaubung}

Die Blätter des Feld-Ahorns sind wie bei allen Ahornen gegenständig angeordnet. Ihre Form ist recht variabel, meistens sind sie 3- bis 5-lappig und bis zur Hälfte der Spreite eingeschnitten, wobei ihre Blattlappen stumpf sind. Die Blätter sind insgesamt deutlich kleiner als die von Berg- und Spitz-Ahorn. Im Austrieb sind sie dunkelrot bis bronzefarben gefärbt und werden

Abb. 4 (oben): Zweig von Acer campestre 'Postelense'.

Abb. 5 (unten): Früchte von Acer campestre.
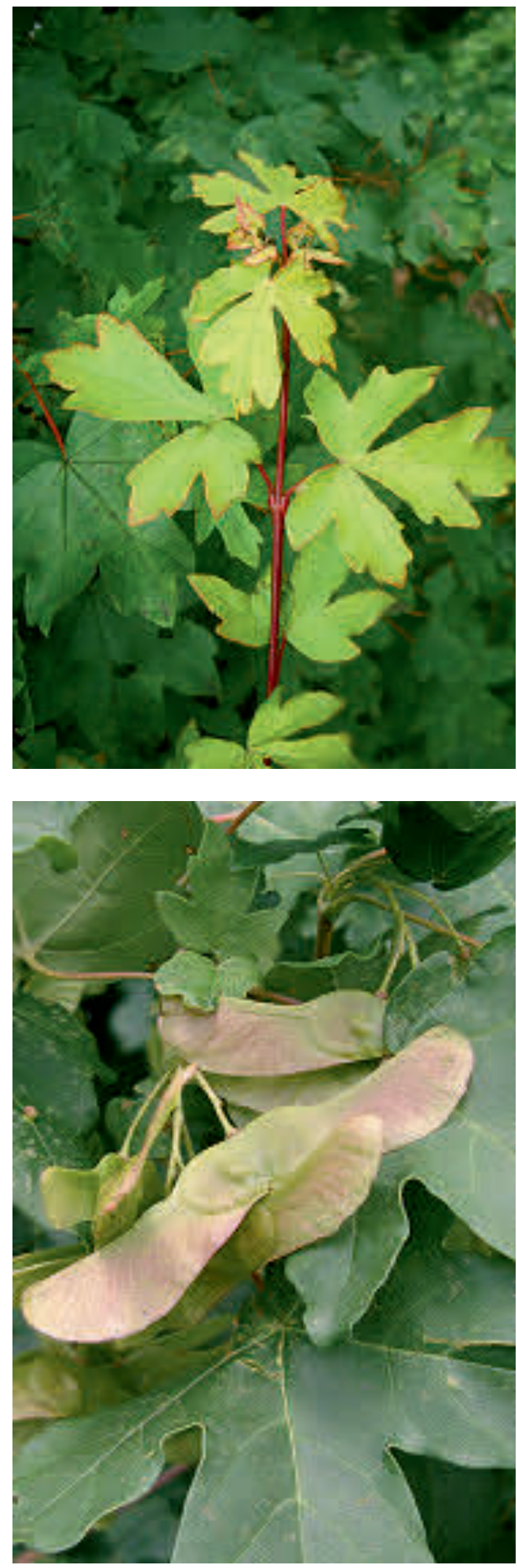


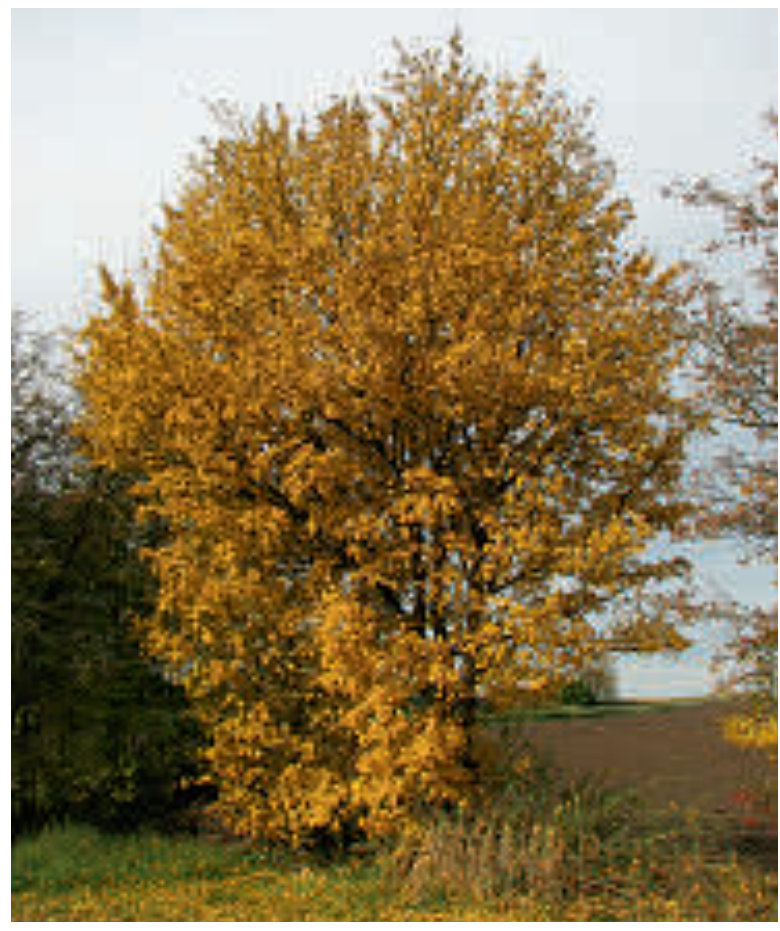

dann mit der Zeit dunkelgrün, im Herbst färben sie sich in ein intensives Gelb bis Goldgelb.

\subsection{Blüten}

Der Feld-Ahorn ist monözisch. Die in aufrechten Doldentrauben angeordneten Blüten öffnen sich ungefähr Ende April bis Mitte Mai. Die lang gestielten Blüten fallen aufgrund ihrer gelblich grünen Farbe zwischen dem ähnlich gefärbten frischen Blattaustrieb oft kaum auf. $\mathrm{Zu}$ einem frühen Entwicklungszeitpunkt der Blüte werden zunächst weibliche und männliche Blütenorgane angelegt. Im weiteren Verlauf findet eine Geschlechterdifferenzierung der Blüten statt. In einem Teil der Blüten werden nur die Staubblätter fertig ausgebildet. Bei anderen entwickeln sich dagegen nur die Fruchtblätter, während die Staubblätter rudimentär bleiben. Bei wieder anderen Blüten entwickeln sich Staub- sowie Fruchtblätter zu voller Größe, weshalb der Anschein einer Zwitterblüte entsteht. Bei diesen scheinbar zwittrigen Blüten öffnen sich die Staubblätter allerdings nicht und setzten dementsprechend auch keinen Pollen frei (Leins \& Erbar 2008). Derartige Blüten sind also funktionell ebenfalls weiblich.

Schon zur Blütezeit sind an den oberständigen Fruchtknoten die beiden Flügel zu erken- nen, aus denen sich später die charakteristischen Fruchtflügel entwickeln. Diese ragen seitlich in die Position von zwei der ursprünglich zehn Staubblätter. An diesen Stellen sind die Staubblätter reduziert, sodass die Blüte insgesamt acht Staubblätter enthält. Zur Verhinderung von Selbstbestäubung entfalten sich männliche und weibliche Blüten nacheinander (Dichogamie), wobei es vorweibliche (protogyne) und vormännliche (protandrische) Pflanzen gibt. Die Blüten werden von Bienen, Hummeln, Fliegen und Käfern besucht.

\subsection{Früchte}

Die Ende September reifenden Früchte sind wie bei allen Ahorn-Arten Spaltfrüchte. Sie bestehen aus zwei Fruchtblättern, die zur Reife entlang der Verwachsungsnaht in zwei einsamige, geflügelte Nussfrüchte zerfallen. Die Fruchtflügel sind beim Feld-Ahorn waagerecht angeordnet. Sie verhelfen der reifen Frucht bzw. nach Zerbrechen der Teilfrucht zu einer propellerartigen Flugbewegung, sodass diese sehr gut durch den Wind ausgebreitet werden kann. Das Nüsschen ist im Vergleich zu anderen Ahorn-Arten relativ flach. Junge Früchte sind zunächst hellgrün, später dunkelgrün bis rötlich und zur Reife bräunlich gefärbt.

\section{Verwendung}

Der Feld-Ahorn ist ein wichtiger Nutzbaum. Das Holz ist dem von Berg-Ahorn (Acer pseudoplatanus) recht ähnlich, unterscheidet sich aber von diesem durch eine dunklere bräunliche oder rötlich weiße Färbung, eine höhere Dichte und das Vorhandensein von Markflecken (SснӥтT et al. 2002). Wegen seiner nicht so massiven Stämme spielt der Feld-Ahorn als Lieferant von Bau- und Möbelholz nur eine geringe Rolle.

Abb. 6 (oben): Wie auch andere Ahornarten färbt sich der Feld-Ahorn im Herbst leuchtend gelb.

Abb. 7 (Seite 15 links): Rasterelektronenmikroskopisches Bild einer Blütenknospe; deutlich zu erkennen sind Staubblätter und Fruchtblatt.

Abb. 8 (Seite 15 rechts): Seitenansicht eines jungen Blütenstandes; rasterelektronenmikroskopische Aufnahme. 


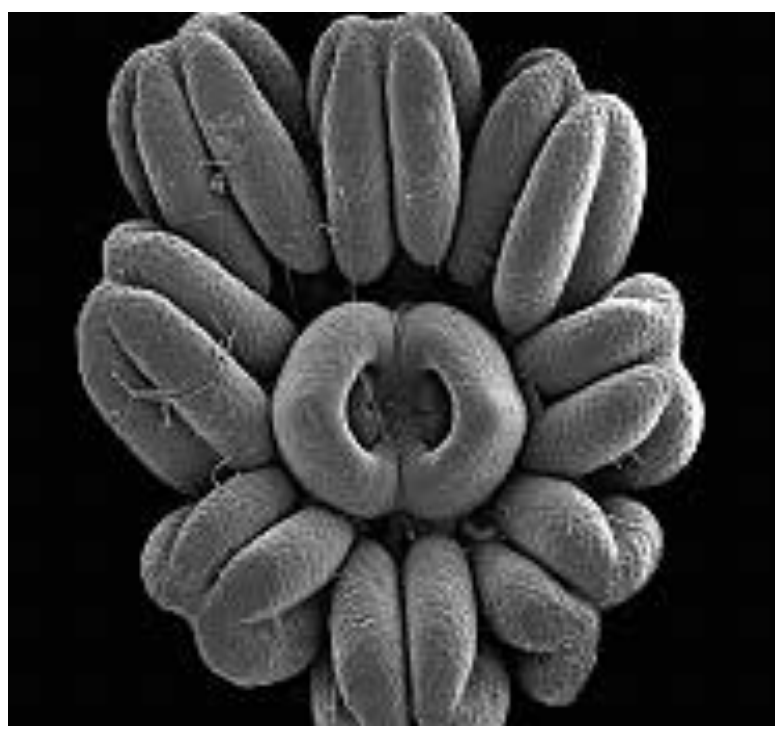

Härte und Zähigkeit des Holzes eignen sich aber für Drechselarbeiten, Schnitzereien und zur Anfertigung von Werkzeugstielen und Musikinstrumenten (z. B. Flöten). Eine größere Bedeutung hat der Feld-Ahorn aufgrund seiner ausgesprochenen Windfestigkeit z. B. in Windschutzhecken. Wegen des intensiven Herzwurzelsystems und der Verträglichkeit gegenüber Überschüttung mit Lockermaterial findet der Feld-Ahorn ebenso Einsatz bei Hangbefestigungen. Auch als Dorf-, Allee- oder Hofbaum sowie zur Verwendung als Formschnitthecke und als wichtiges Vogelnähr- und Nistgehölz ist er beliebt. Das Regenerationsvermögen nach starkem Rückschnitt durch Stockausschlag ist sehr hoch, weshalb der Feld-Ahorn zur Nieder- und Mittelwaldwirtschaft sowie zur Nebenbestandsbildung geeignet ist (Кснüтt et al. 2002). Das Falllaub kann als Viehfutter verwendet werden.

Besonders die Zuchtsorten des Feld-Ahorns sind wichtige Ziergehölze. Im Handel werden zahlreiche gärtnerische Selektionen angeboten, die sich von der Stammform durch abweichende Wuchsformen und Belaubungsmerkmale unterscheiden. Acer campestre 'Nanum' hat eine dicht verzweigte, mehr oder weniger rundliche Krone. Die Sorten 'Eastleigh Weeping' und 'Green Weeping' sind Hängeformen, während Sorten wie 'Elsrijk', 'Queen Elizabeth', 'Schwerinii' und 'Zorgvlied' eine straff aufrechte bis mehr oder weniger breit säulenförmige Krone aufweisen. Sorten mit abweichenden Be-

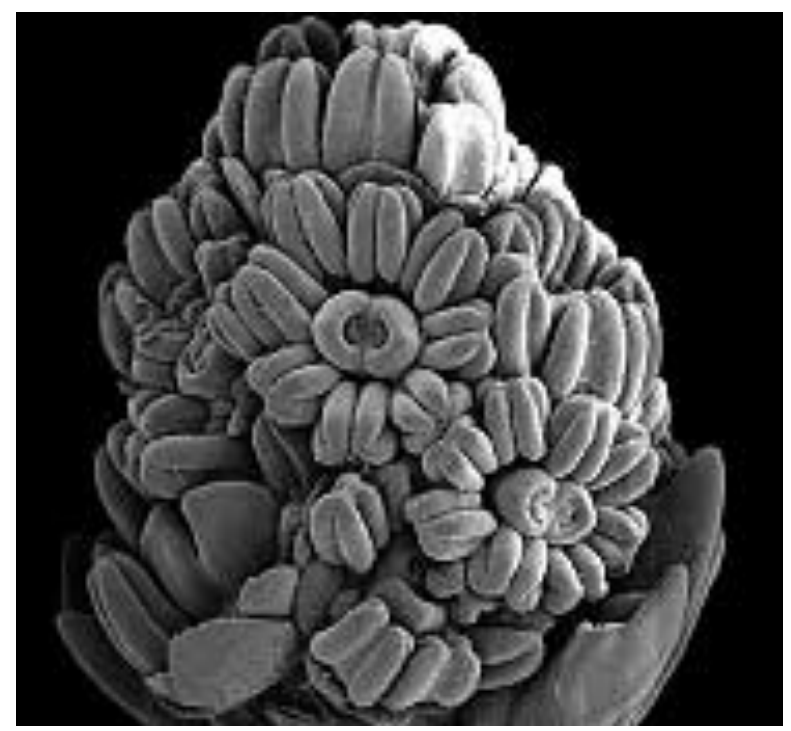

laubungsmerkmalen sind z. B. der sehr kleinblättrige $A$. campestre 'Microphyllum' oder der auffallend großblättrige 'Macrophyllum'. Acer campestre 'Red Shine' oder 'Royal Ruby' weisen einen intensiven roten Austrieb auf. Das Sommerlaub ist hingegen dunkel olivgrün. Weißbuntes Laub findet man bei $A$. campestre 'Carnival' und 'Pulverulentum' (intensiv weiß gesprenkelt). Acer campestre 'Postelense' ist im Austrieb goldgelb, später gelbgrün gefärbt. Die weiß- und gelblaubigen Kultivare benötigen einen lichtschattigen Standort, da die Blätter auf vollsonnigen Standorten zu Schäden durch Sonnenbrand neigen.

\section{Literatur}

Kiermeier, P. 1993: Wildgehölze des mitteleuropäischen Raumes, BdB-Handbuch, Teil VIII, 5. Aufl. - Pinneberg. Leins, P. \& Erbar, C. 2008: Blüte und Frucht. - Stuttgart.

Pirc, H. 1994: Ahorne. - Stuttgart.

Prasse, R., Ristow, M., Klemm; G., Machatzi, B., Raus, T., Scholz, H., Stohr, G., Sukopp, H. \& Zimmermann, F. 2001: Liste der wildwachsenden Gefäßpflanzen des Landes Berlin mit Roter Liste. - Berlin. Schütт, P., Schuck, H. J. \& Sтімm, B. 2002: Lexikon der Baum- und Straucharten. - Hamburg.

Van Gelderen, D. M., de Jong, P. C. \& Oterdoom, H. J. 1994: Maples of the world. - Portland.

Van Gelderen, C. J. \& Van Gelderen, D. M. 1999: Maples for gardens, a color encyclopedia. - Portland.

\section{Internetseite}

Stevens, P. F. 2014: Angiosperm Phylogeny Website, Version 13. - http://www.-mobot.org/MOBOT/research/ APweb/ [30.12.2014]. 